Research Article

\title{
Analysis of the Components in Moxa Smoke by GC-MS and Preliminary Discussion on Its Toxicity and Side Effects
}

\author{
Xiaoyu Xu $\mathbb{D}^{1,2}$ Si Shan $\mathbb{D}^{1,2}$, Wenlei Wang $\mathbb{D}^{1,2}$ and Hongning Liu ${ }^{1,2}$ \\ ${ }^{1}$ Jiangxi Province Key Laboratory of TCM Etiopathogenisis, Jiangxi University of Traditional Chinese Medicine, Nanchang, \\ Jiangxi 330004, China \\ ${ }^{2}$ Research Center for Differentiation and Development of TCM Basic Theory, Jiangxi University of Traditional Chinese Medicine, \\ Nanchang, Jiangxi 330004, China \\ Correspondence should be addressed to Si Shan; shansi1987@163.com
}

Received 20 May 2020; Revised 21 September 2020; Accepted 1 October 2020; Published 31 October 2020

Academic Editor: Luísa Zuravski

Copyright ( $\odot 2020$ Xiaoyu Xu et al. This is an open access article distributed under the Creative Commons Attribution License, which permits unrestricted use, distribution, and reproduction in any medium, provided the original work is properly cited.

\begin{abstract}
Moxibustion plays an important role in the prevention and treatment of diseases and the promotion of human health. In this study, the components in moxa smoke from Jiangxi Poai Biotechnology Co., Ltd., namely, Qing moxa sticks, were absorbed by five solvents (cyclohexane, ethyl acetate, $n$-butanol, anhydrous ethanol, and water) and identified by gas chromatography-mass spectrometry. The identification results of the smoke from the Qing moxa sticks that was absorbed in liquid are as follows: a total of 294 compounds were identified, including 139 in cyclohexane, 145 in ethyl acetate, 60 in $n$-butanol, 89 in anhydrous ethanol, and 77 in water, and of those, 112 toxic compounds were identified. Furthermore, Ingenuity Pathway Analysis software and the PubChem database were successfully applied to analyze the toxic compounds. There were 812 target proteins related to the toxic components, 25 molecular networks, and 54 biological pathways. The results showed that the toxic compounds of moxa smoke may have some side effects on the heart, liver, and kidney of humans. This study revealed that the components of moxa smoke are complex and diverse. Due to the findings of toxic compounds in moxa smoke, we recommend that moxibustion rooms should be equipped with ventilation equipment or enough artificial ventilation to ensure the health of patients and practitioners.
\end{abstract}

\section{Introduction}

Moxibustion is an important part of clinical treatment in traditional Chinese medicine. In moxibustion, wormwood or other drugs are used to place acupoints or pain points on the body surface for warming meridians and stimulating acupuncture points [1]. As people pay more attention to health, the use of moxibustion to treat diseases in China and other Asian countries is growing [2]. Heat and moxa smoke are produced during moxibustion. The heat of moxibustion has the function of assisting Yang Qi, lifting subsidence, and solidifying. Recent studies have shown that moxa smoke also has antibacterial, antitumor, antiviral, anti-inflammatory, and air purification functions [3-7]. Ancient books on Chinese Medicine contain records of the use of moxa smoke in the treatment of irritable bowel syndrome [8], inflammatory bowel disease [9], and neurological symptoms [10].
Additionally, the antioxidants in moxa smoke play an antiaging role through the penetration of heat [11].

However, some patients feel uncomfortable during moxibustion and can even have noticeable adverse reactions, such as watery eyes and coughing, which has caused people to question the safety of moxa smoke $[12,13]$. Some studies have shown that there were harmful components such as monoaromatic hydrocarbons and formaldehyde in moxa smoke [14-16]. The inhalation of these substances induced eustachian tube irritation, throat itching, eye pain, tonsil swelling, and other toxic effects [12]. Therefore, it is very important to determine the toxic compounds in moxa smoke.

The aim of the present study was to analyze the components in Qing moxa smoke based on enrichment with five solvents. A set of smoke absorption devices were designed with cyclohexane, ethyl acetate, $n$-butanol, anhydrous 
ethanol, and water as absorbents with the help of an extraction pump to concentrate the moxa smoke in the solvents. The benefits of this device for enrichment of moxa smoke include: (1) moxa sticks can burn completely in the air to avoid incomplete combustion; (2) the devices can detect as many compounds as possible by increasing the concentration of moxa smoke; and (3) the use of different polar solvents can provide reference for the absorption and treatment of moxa smoke. Then, the toxic compounds were queried by the Comparative Toxicogenomics Database (CTD) $[17,18]$. In addition, we aimed to estimate the toxic compounds in moxa smoke that would have an impact on the human body by applying Ingenuity Pathway Analysis (IPA) software and the PubChem database to provide an experimental basis for the safety evaluation of moxa smoke $[19,20]$.

\section{Materials and Methods}

2.1. Materials. We followed the steps outlined in our patent, "A method of using Terahertz Wave to detect the quality of moxa column," patent number: ZL 20201 0000161.6, which are as follows: (1) sample placement; (2) determination of the background value; (3) measurement of the terahertz wave energy at different bands of the combustion column; (4) data processing; and (5) column quality judgment. If the terahertz wave intensity of each band is stronger than others and the waveform slightly changes, the quality is better. The results revealed which Qing moxa stick had the best quality, and that one was selected for smoke enrichment analysis [21]. Qing moxa sticks $(18 \times 27 \pm 1 \mathrm{~mm}$, Jiangxi Poai Biotechnology Co., Ltd., Poyang, China), which are widely used by the Chinese population, were used in this study. Moxa sticks were encased in Artemisia argyi (Chinese mugwort) floss, which was made of dried $A$. argyi leaves. The Qing moxa sticks were produced with a $10: 1$ ratio, which means that $10 \mathrm{~kg}$ of dried A. argyi leaves were processed into $1 \mathrm{~kg}$ of moxa floss. Analytical grade cyclohexane, ethyl acetate, $n$-butanol, and ethanol were all purchased from Guangdong Xilong Science Co., Ltd., and used as received.

2.2. Sample Preparation. A set of smoke absorption devices was designed as shown in Figure 1. With $1000 \mathrm{~mL}$ cyclohexane, ethyl acetate, $n$-butanol, anhydrous ethanol, or water as the solvent, 50 moxa sticks were burned in the air until combustion was complete. During the combustion process, the air pump control combustion speed was adjusted such that the blank flask did not fill with white smoke, so that the solvent fully absorbed the moxa smoke. The glass ball in the absorption flask had holes in it to reduce the production of bubbles and prevent the solvent from escaping. The absorption solution was emptied from the absorption flask, filtered with a $0.22 \mu \mathrm{m}$ microporous membrane, and $2 \mathrm{~mL}$ of each solution was added into the sample bottle for gas chromatography-mass spectroscopy (GC-MS) analysis.
2.3. GC-MS Analysis. An Agilent Technologies 7890 GC system (Agilent Technologies Inc., Palo Alto, CA, USA) coupled with an Agilent Technologies 5975 mass spectrometer (Agilent Technologies Inc.) was used for moxa smoke analysis. A HP-5MS capillary column $(30 \mathrm{~m} \times 0.25 \mathrm{~mm} \times 0.25 \mu \mathrm{m})$ was used to separate compounds. High-purity helium was applied as the carrier gas. The following conditions were used: column flow rate: $1.0 \mathrm{~mL} / \mathrm{min}$; split injection, split ratio: $100: 1$; injection volume: $1 \mu \mathrm{L}$; and injection port temperature: $250^{\circ} \mathrm{C}$. The temperature procedure was as follows: $0-3 \mathrm{~min}, 40-40^{\circ} \mathrm{C}$; 3-39 min, $40-220^{\circ} \mathrm{C}$; $39-43 \mathrm{~min}, 220-220^{\circ} \mathrm{C}$; $43-49 \mathrm{~min}$, $220-280^{\circ} \mathrm{C}$; and $49-50 \mathrm{~min}, 280-280^{\circ} \mathrm{C}$.

The MS working conditions were as follows: the electron ionization energy was $70 \mathrm{eV}$, the full-scan acquisition was used in the range of $50-650 \mathrm{~m} / \mathrm{z}$, the ion source temperature was $230^{\circ} \mathrm{C}$, the transmission ion temperature was $280^{\circ} \mathrm{C}$, and the four-stage pole temperature was $150^{\circ} \mathrm{C}$. The identification of each peak in the total ion flow chromatogram was automatically retrieved from the National Institute of Standards and Technology (NIST) 11.L as the standard mass spectrometry database and verified with standard mass spectrometry. Some components were confirmed with the retention value of a standard sample. The identified components were semiquantified by comparing the peak area of each component with the total peak area, and the relative percentage of components was calculated by the peak area normalization method.

2.4. Network Toxicological Analysis. The compounds identified by the NIST 11.L were then queried for related toxicity through the CTD database (https://ctdbase.org/about/). Then, the molecular information corresponding to the toxic compounds of moxa smoke was obtained from the PubChem database (http://pubchem.ncbi.nlm.nih.gov/) [22]. In addition, the Swiss Target Prediction database (http://www. swisstargetprediction.ch/) was used to predict toxic compounds relevant targets, and exporting Uniprot ID. Next, the molecular networks of toxic compound target proteins and its biological pathways were constructed by IPA software (Qiagen, Redwood City, CA, USA).

\section{Results}

3.1. Total Ion Chromatogram. The total ion chromatograms (TIC) of moxa smoke from solvents by GC-MS are shown in Figure $2[23,24]$. As shown in Figure 2, the compounds in moxa smoke were detected within $40 \mathrm{~min}$.

3.2. GC-MS Analysis Results. A total of 294 compounds, including 139 in cyclohexane, 145 in ethyl acetate, 60 in $n$ butanol, 89 in anhydrous ethanol, and 77 in water were found and identified in Qing moxa smoke. As shown in Tables 1-5, only 52 unique compounds were detected in cyclohexane smoke absorption liquid, 57 in ethyl acetate, 10 in $n$-butanol, 17 in anhydrous ethanol, and 47 in water, and other components were identified in more than one solvent. Toluene, pyridine, 2-methylpyridine, 2-methyl-2-cyclopenten-1-one, 


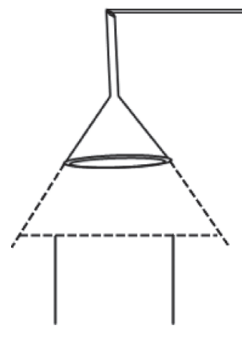

(1)

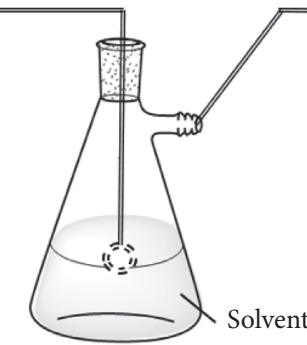

(2)

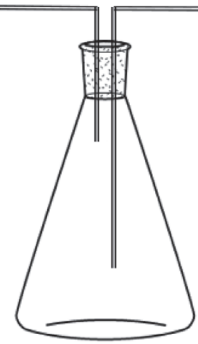

(3)

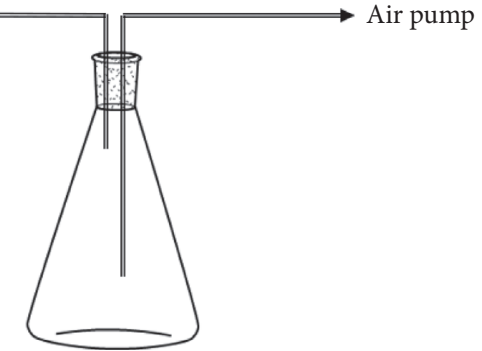

(4)

Figure 1: A smoke absorption device. (1) Smoke hood. (2) Absorption flask. (3) Blank flask. (4) Buffer flask.

2-furanmethanol, 2-acetylfuran, phenol, eucalyptol, o-cresol, indole, and biphenyl were detected in all five solvents and are shown in Figure 3, but the same components had different concentrations in different solvents. This shows that the components of moxa smoke were absorbed differently by different polar solvents.

As shown in Figure 3, the common components from moxa smoke in the five solvents included toluene $(0.650 \%-$ $3.872 \%)$, pyridine $(0.137 \%-2.847 \%), \quad 2$-methylpyridine $(0.267 \%-1.878 \%), 2$-methyl-2-cyclopenten-1-one $(0.412 \%$ $1.649 \%)$, 2 -furanmethanol $(0.526 \%-1.320 \%)$, 2 -acetylfuran $(0.266 \%-1.092 \%)$, phenol $(2.686 \%-5.405 \%)$, eucalyptol $(1.037 \%-1.605 \%), \quad$ o-cresol $\quad(0.661 \%-1.419 \%), \quad$ indole $(0.780 \%-1.257 \%)$, and biphenyl (0.179\%-0.338\%). Among the above common components, the relative contents of phenol were more than $2 \%$ in all solvents. Phenol is a corrosive compound that is a strong irritant, which can lead to acute poisoning, skin ulcers, and tissue burns and can even be life-threatening $[25,26]$. However, the amount of harmful substances produced by moxibustion will dictate the negative impact on the human body, and the duration of exposure to a moxa fume environment will determine if damage is caused to the body. There is no unified answer to these questions, which requires a large amount of case analysis and clinical trials.

3.3. Toxic Compounds of Moxa Smoke. The toxicity of compounds was determined based on the CTD database (https://ctdbase.org/about/), which provided abundant toxicological information for researchers. Among the 294 compounds detected in the moxa smoke absorption liquid, 112 compounds were confirmed to be toxic. Further study is needed to explore the toxicity of the 112 compounds. Table 6 provides details of the 112 toxic compounds.

3.4. Targets of Toxic Compounds. Through the PubChem database (http://pubchem.ncbi.nlm.nih.gov/), molecular information for the 112 toxic compounds in moxa smoke was identified, and the corresponding number of "Canonical SMILES" was obtained. Then, using the Swiss Target Prediction database (http://www.swisstargetprediction.ch/) to predict the 112 relevant targets of the toxic compounds, the UniProt ID was exported. In addition, the UniProt ID was analyzed with IPA software to obtain the targets of toxic compounds. There were 812 targets for the toxic compounds in moxa smoke, compared to 810 identified with the IPA database.

3.5. Molecular Networks of Toxic Compounds. The UniProt IDs of the 810 target proteins of the 112 toxic compounds were imported into the IPA bioanalysis software. Under the "tox analysis" module, IPA was used to construct the molecular networks of target proteins. A total of 25 molecular networks were constructed for 112 toxic compounds, with a maximum score of 43, as shown in Figure 4. The results showed that these target proteins were related to cell signal transduction, nucleic acid metabolism, inflammatory response, organ damage, and cell apoptosis. Therefore, this can be used to frame a correlation study on moxa smoke.

3.6. Biological Pathways of Toxic Compounds. Using the "tox analysis" module in the IPA software, a total of 54 biological pathways were found for the 112 toxic compounds. The main biological pathways of the toxic compounds from moxa smoke included cardiotoxicity, hepatotoxicity, and nephrotoxicity. Consequently, the toxic compounds of moxa smoke may have some side effects on the human heart, liver, and kidneys. A heat map of the biological pathway of toxic compounds is shown in Figure 5. According to it, the pathway with the highest $-\log$ ( $p$ value) was cardiac arteriopathy, which was classified as cardiotoxicity, with a value of 79.429. Drug target molecules acting on this pathway include ABCB1, ABCC8, ACE, ADORA1, ADORA2A, ADORA2B, ADORA3, ADRA2A, ADRA2B, ADRA2C, ADRB1, ADRB2, ADRB3, ALDH5A1, ALOX5AP, AR, ASIC3, CA1, CA12, CA13, CA14, CA2, CA3, CA4, CA5A, CA5B, CA6, CA7, CA9, CACNA2D1, CETP, CNR1, CYP2C19, CYP2C9, DPP4, ESR1, ESR2, F10, F2, F2R, FADS1, FKBR1A, FLT1, FLT4, GAA, GABRA1*, GABRA2 $*$, GABRA3 $*$, GABRA5*, GABRB2 $*$, GABRB3*, GABRG2*, GLP1R, GLRA1, GRIA4, HCAR2, HMGCR, HRH2, HTT, ICAM1, INSR, ITGAL, ITGB2, KCNA5, KCNJ11, KDM1A, KDR, MTNR1A, MTNR1B, MTOR, NOS3, NPC1L1, NR3C1, NR3C2, OPRD1, OPRK1, OPRM1, PDE10A, PDE11A, PDE3A, PDE3B, PDE4A, PDE4B, PDE4C, PDE4D, PDE5A, PDE7A, PDE7B, PGR, PLA2G2A, PLA2G7, PLG, PPARA, PPARG, PRCP, PRKCH, PTGER1, PTGER2, PTGER3, PTGER4, PTGIR, PTGS1, PTGS2, RHOA, S1PR1, SCARB1, SCN10A, SCNSA, 


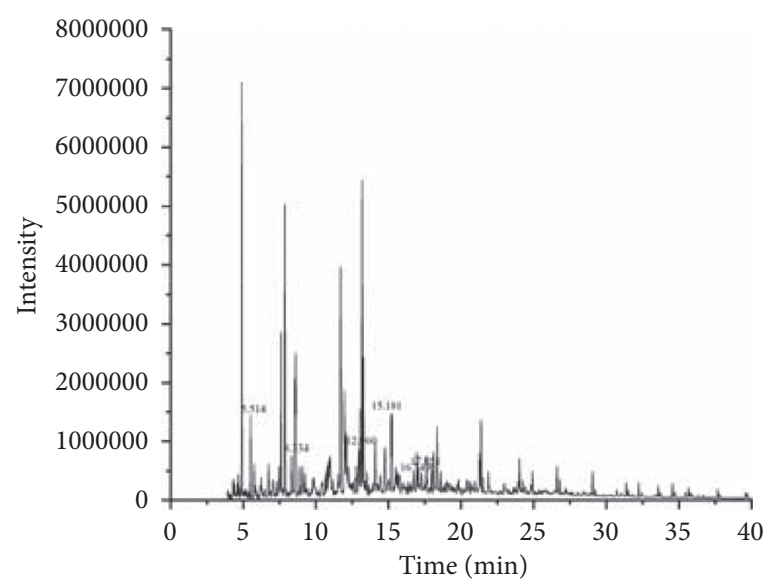

(a)

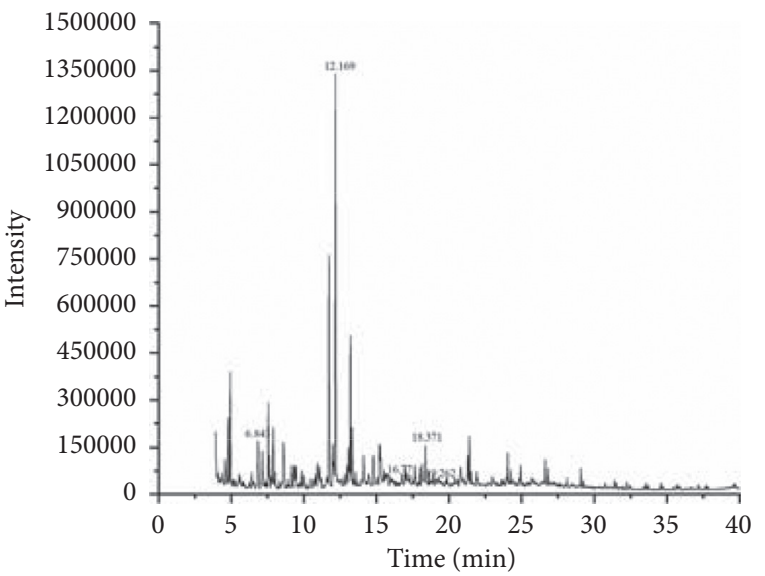

(c)

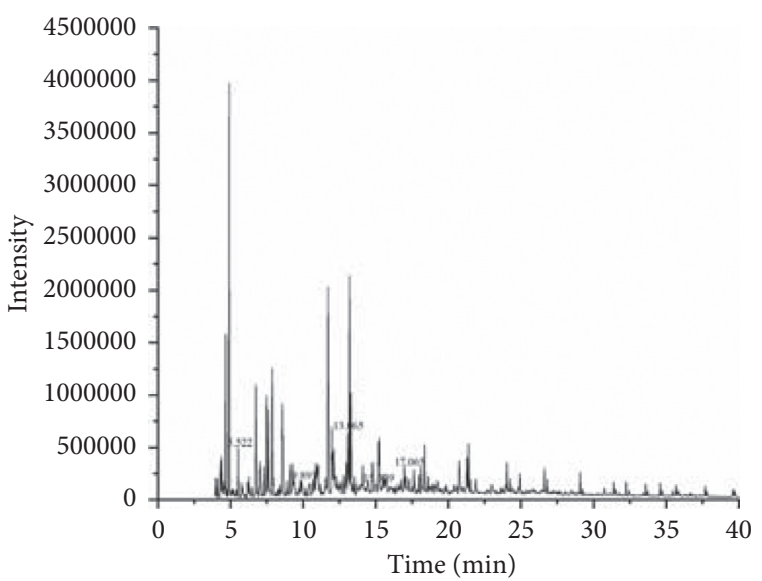

(b)

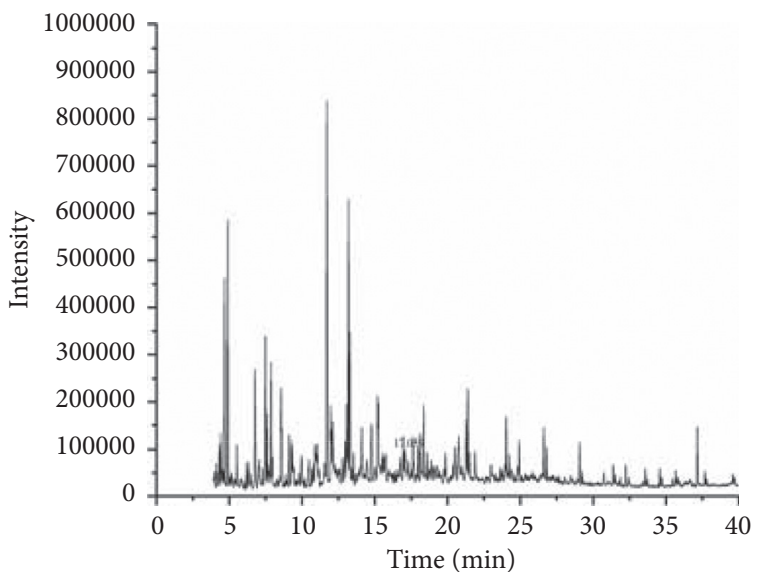

(d)

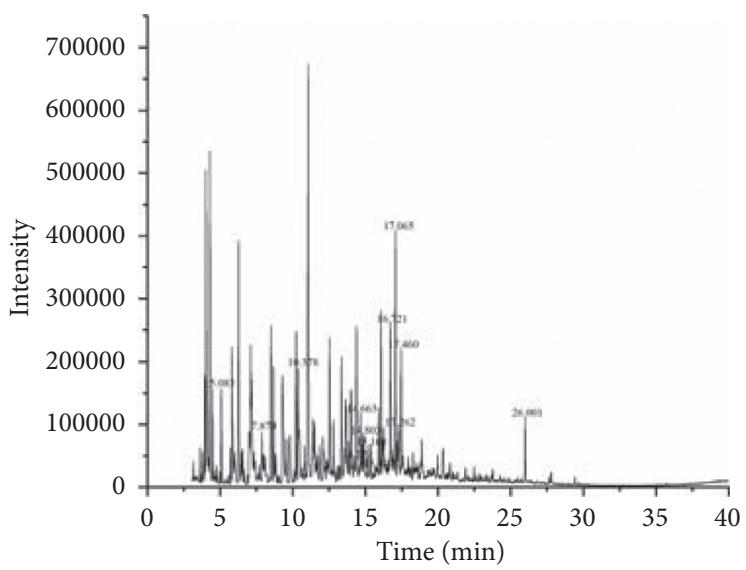

(e)

Figure 2: Total ion chromatograms of five solvents by GC-MS. (a) Cyclohexane. (b) Ethyl acetate. (c) $n$-Butanol. (d) Anhydrous ethanol. (e) Water. Only compounds unique to each solvent with a relative content greater than $0.5 \%$ are tagged in the figure.

SCN9A, SELE, SERPINE1, SLC6A4, SOAT1, TBXA2R, TERT, TLR4, TNF, TNNT2 $*$, TSPO, TUBB1, TUBB3, VDR, VEGFA, and XDH. This also guides the development of follow-up toxicology experiments and research on the effects of moxa smoke on the organs of Sprague Dawley rats.

\section{Discussion}

GC-MS was applied to study the compounds in moxa smoke absorbed in five different polar solvents from Qing moxa sticks. This study found that a total of 294 compounds were 
TABLE 1: Relative content (\%) of unique compounds in cyclohexane.

\begin{tabular}{|c|c|c|c|c|}
\hline No. & Rt (min) & Compound & Retention index & Relative content (\%) \\
\hline 1 & 4.038 & Bicyclo[4.1.0]hept-2-ene & 706 & 0.033 \\
\hline 2 & 4.602 & 4-Methyl-1,4-hexadiene & 737 & 0.175 \\
\hline 3 & 4.978 & 1-Methylcyclohexene & 757 & 0.078 \\
\hline 4 & 5.093 & 1,3,5-Heptatriene & 763 & 0.115 \\
\hline 5 & 5.290 & 1,7-Octadiene & 774 & 0.048 \\
\hline 6 & 5.400 & 2-Methyl-1-heptene & 780 & 0.105 \\
\hline 7 & 5.514 & 1-Octene & 786 & 0.693 \\
\hline 8 & 5.947 & (E)-2-Octene & 806 & 0.086 \\
\hline 9 & 6.458 & 1,3-Dimethyl cyclohexene & 822 & 0.222 \\
\hline 10 & 6.647 & (E,E,E)-2,4,6-Octatriene & 828 & 0.027 \\
\hline 11 & 7.111 & 5,6-Dimethyl-1,3-cyclohexadiene & 843 & 0.040 \\
\hline 12 & 7.749 & 3-Methylenecycloheptene & 863 & 0.146 \\
\hline 13 & 8.060 & $(1 \mathrm{Z}, 2 \mathrm{Z})-1,2-\mathrm{Di}$ (ethylidene)cyclobutane & 873 & 0.094 \\
\hline 14 & 8.334 & Cyclohexanol & 882 & 0.703 \\
\hline 15 & 9.025 & 2-Ethylpyridine & 904 & 0.095 \\
\hline 16 & 10.064 & 2-Methyl-1-octen-3-yne & 935 & 0.105 \\
\hline 17 & 10.166 & 1-Methylcycloheptene & 938 & 0.141 \\
\hline 18 & 11.164 & Mesitylene & 968 & 0.307 \\
\hline 19 & 11.629 & Alpha-methyl styrene & 981 & 0.176 \\
\hline 20 & 12.372 & Gamma-terpinene & 1004 & 0.227 \\
\hline 21 & 12.960 & 1,2,4-Trimethylbenzene & 1022 & 0.866 \\
\hline 22 & 13.395 & Trans-beta-methyl styrene & 1035 & 0.371 \\
\hline 23 & 14.973 & 1-Phenyl-2-butene & 1083 & 0.228 \\
\hline 24 & 15.181 & 1-Methyl-4-(prop-1-en-2-yl)benzene & 1090 & 1.054 \\
\hline 25 & 15.901 & Cosmene & 1112 & 0.163 \\
\hline 26 & 16.210 & 2,4-Dimethylstyrene & 1122 & 0.156 \\
\hline 27 & 16.305 & 1-Phenyl-1-butene & 1125 & 0.336 \\
\hline 28 & 16.448 & 1-Allyl-2-methylbenzene & 1130 & 0.223 \\
\hline 29 & 16.749 & Phenyl acetonitrile & 1140 & 0.610 \\
\hline 30 & 17.051 & 2,3-Dimethylphenol & 1149 & 0.687 \\
\hline 31 & 17.155 & 1,2,3,4-Tetramethylbenzene & 1153 & 0.203 \\
\hline 32 & 17.510 & 1,1a,6,6a-Tetrahydrocycloprop $[\mathrm{a}]$ indene & 1164 & 0.258 \\
\hline 33 & 18.241 & 1,2-Dimethylindan & 1188 & 0.200 \\
\hline 34 & 19.970 & 4-Methylindole & 1247 & 0.200 \\
\hline 35 & 20.792 & 7H-Benzocycloheptene & 1275 & 0.200 \\
\hline 36 & 21.045 & 2-(2-Hydroxyphenyl)buta-1,3-diene & 1284 & 0.168 \\
\hline 37 & 22.396 & 1H-Indene,2,3-dihydro-1,1,3-trimethyl & 1332 & 0.226 \\
\hline 38 & 23.401 & 5-Methylindole & 1369 & 0.099 \\
\hline 39 & 23.527 & 1,8-Cyclotetradecadiyne & 1374 & 0.108 \\
\hline 40 & 24.816 & 1,4-Dimethylnaphthalene & 1422 & 0.227 \\
\hline 41 & 25.073 & 1,4,5-Trimethylnaphthalene & 1432 & 0.142 \\
\hline 42 & 26.434 & 2,4,6-Trimethylbenzonitrile & 1485 & 0.137 \\
\hline 43 & 27.199 & 1-Phenylpyridin-2-one & 1516 & 0.186 \\
\hline 44 & 28.473 & 2,4-Dimethoxyacetophenone & 1568 & 0.234 \\
\hline 45 & 28.910 & Spathulenol & 1586 & 0.163 \\
\hline 46 & 29.355 & 2-Methylbiphenyl & 1605 & 0.114 \\
\hline 47 & 30.735 & $(+)-\gamma$-Gurjunene & 1665 & 0.217 \\
\hline 48 & 33.434 & 9-Methylene-9H-fluorene & 1786 & 0.103 \\
\hline 49 & 34.460 & 3,7,11,15-Tetramethyl-2-hexadecene & 1835 & 0.062 \\
\hline 50 & 34.710 & 2,6,10,14-Tetramethyl-2-hexadecene & 1847 & 0.135 \\
\hline 51 & 35.671 & 1-Nonadecene & 1893 & 0.163 \\
\hline 52 & 37.669 & E-15-Heptadecenal & 1993 & 0.123 \\
\hline
\end{tabular}

identified, including 139 in cyclohexane, 145 in ethyl acetate, 60 in $n$-butanol, 89 in anhydrous ethanol, 77 in water, and 11 in all five polar solvents. Among the 294 compounds detected in the moxa smoke absorption liquid, 112 compounds were confirmed to be toxic. With the "tox analysis" module, IPA was used to construct molecular networks of target proteins. The results showed that these target proteins were related to cell signal transduction, nucleic acid metabolism, inflammatory response, organ damage, and cell apoptosis. At the same time, the main biological pathways of the toxic compounds from moxa smoke included cardiotoxicity, hepatotoxicity, and nephrotoxicity. The safety of smoke has 
TABLE 2: Relative content (\%) of unique compounds in ethyl acetate.

\begin{tabular}{|c|c|c|c|c|}
\hline No. & Rt (min) & Compound & Retention index & Relative content (\%) \\
\hline 1 & 4.106 & 3-Methyl-butanenitrile & 710 & 0.159 \\
\hline 2 & 4.535 & Dimethyl aminoacetonitrile & 733 & 0.191 \\
\hline 3 & 4.603 & 2,4-Dimethyl-1,3-pentadiene & 737 & 0.152 \\
\hline 4 & 5.236 & 3-Methylenecyclohexene & 771 & 0.095 \\
\hline 5 & 5.300 & Cyclooctene & 775 & 0.030 \\
\hline 6 & 5.522 & 2-Octene & 787 & 0.557 \\
\hline 7 & 5.837 & 2,3-Dimethyl-1,4-hexadiene & 802 & 0.120 \\
\hline 8 & 5.957 & (Z)-2-Octene & 806 & 0.044 \\
\hline 9 & 6.462 & Pyrazine, methyl & 822 & 0.208 \\
\hline 10 & 6.869 & 2,5-Dimethylpyrrole & 835 & 0.088 \\
\hline 11 & 8.073 & 1,4-Dimethylenecyclohexane & 874 & 0.051 \\
\hline 12 & 8.411 & 2,3-Dimethylpyridine & 885 & 0.166 \\
\hline 13 & 9.820 & 3-Ethyl-1H-pyrrole & 928 & 0.295 \\
\hline 14 & 9.893 & 3,4-Dimethylpyridine & 930 & 0.509 \\
\hline 15 & 10.078 & 2-Ethyl-5,5-dimethyl-1,3-cyclopentadien & 935 & 0.082 \\
\hline 16 & 10.480 & 2-Methylborazine & 947 & 0.250 \\
\hline 17 & 11.291 & 2,5-Cyclooctadien-1-one & 971 & 0.115 \\
\hline 18 & 11.406 & Benzene & 975 & 0.060 \\
\hline 19 & 11.503 & Aniline & 978 & 0.183 \\
\hline 20 & 12.166 & 3-Methylstyrene & 997 & 0.337 \\
\hline 21 & 12.385 & Alpha-phellandrene & 1004 & 0.151 \\
\hline 22 & 12.591 & 2-Ethyl-4-methyl-1H-pyrrole & 1010 & 0.295 \\
\hline 23 & 13.065 & o-Cymene & 1025 & 1.118 \\
\hline 24 & 13.402 & Allylbenzene & 1035 & 0.309 \\
\hline 25 & 15.039 & 3-Ethyl-o-xylene & 1085 & 0.593 \\
\hline 26 & 15.620 & 7-Methylbenzofuran & 1103 & 0.383 \\
\hline 27 & 15.921 & Azulene & 1113 & 0.406 \\
\hline 28 & 16.454 & 4-Allyltoluene & 1130 & 0.171 \\
\hline 29 & 16.762 & 3-Ethynylaniline & 1140 & 0.367 \\
\hline 30 & 17.065 & 3-Methyl-1H-indene & 1150 & 0.557 \\
\hline 31 & 17.163 & 1,2,3,4-Tetramethylfulven & 1153 & 0.138 \\
\hline 32 & 17.523 & 1,4-Dihydronaphthalene & 1165 & 0.214 \\
\hline 33 & 18.254 & 1-Methyl-3-(1-methyl-2-propenyl)benzene & 1188 & 0.188 \\
\hline 34 & 18.501 & Dihydrocarveol & 1196 & 0.140 \\
\hline 35 & 18.696 & Catechol & 1203 & 0.223 \\
\hline 36 & 19.034 & 2,6-Dimethylundecane & 1215 & 0.195 \\
\hline 37 & 19.250 & (E)-Cinnamaldehyde & 1222 & 0.391 \\
\hline 38 & 19.355 & Cyclododecene & 1226 & 0.166 \\
\hline 39 & 19.758 & Isoquinoline & 1239 & 0.210 \\
\hline 40 & 19.983 & 3-Methylindolizine & 1247 & 0.172 \\
\hline 41 & 21.057 & 1,11-Dodecadiene & 1284 & 0.162 \\
\hline 42 & 22.733 & 2-Methylhydroquinone & 1345 & 0.174 \\
\hline 43 & 22.816 & Naphthalene, 1,2,3,4-tetrahydro-1, 1-dimethyl & 1348 & 0.125 \\
\hline 44 & 23.825 & 2-Methyl-5-(1-methylethenyl)-cyclohexanone & 1385 & 0.131 \\
\hline 45 & 23.955 & 3-Methylindole & 1389 & 0.257 \\
\hline 46 & 24.827 & 1,3-Dimethylnaphthalene & 1423 & 0.205 \\
\hline 47 & 24.930 & 1,6-Dimethylnaphthalene & 1427 & 0.460 \\
\hline 48 & 25.761 & 2-Phenyl-1,3-cyclohexadien & 1459 & 0.104 \\
\hline 49 & 28.833 & Phenylephrine & 1583 & 0.020 \\
\hline 50 & 31.836 & Thiazolo[5,4-f] quinolin & 1713 & 0.158 \\
\hline 51 & 32.239 & 1,1,2-Trimethylcycloundecane & 1732 & 0.250 \\
\hline 52 & 33.442 & Phenanthrene & 1787 & 0.116 \\
\hline 53 & 34.719 & 3,7,11,15-Tetramethyl-2-hexadecene & 1847 & 0.146 \\
\hline 54 & 35.480 & (S)-6,6-Dimethyl-2-azaspiro[4.4]non-1-ene & 1884 & 0.158 \\
\hline 55 & 37.681 & 3-Icosene & 1994 & 0.179 \\
\hline 56 & 39.610 & 10-Heneicosene $(c, t)$ & 2093 & 0.140 \\
\hline 57 & 39.729 & Heneicosane & 2100 & 0.110 \\
\hline
\end{tabular}


TABLE 3: Relative content (\%) of unique compounds in $n$-butanol.

\begin{tabular}{lcccc}
\hline No. & Rt $(\mathrm{min})$ & Compound & Retention index & Relative content (\%) \\
\hline 1 & 6.843 & 3-Furaldehyde & 834 & 1.167 \\
2 & 9.850 & 3-Methylheptan-4-one & 928 & 0.377 \\
3 & 9.998 & 2,4-Dimethylpyridine & 933 & 0.457 \\
4 & 10.819 & Limonene & 997 & 0.442 \\
5 & 12.169 & Butyl butyrate & 1126 & 6.894 \\
6 & 16.330 & (E)-1-Phenyl-1-butene & 1140 & 0.273 \\
7 & 16.771 & Benzyl(methylidyne)azanium & 1192 & 0.559 \\
8 & 18.371 & cis-2-dodecene & 1222 & 1.064 \\
9 & 19.262 & Tricyclo[3.3.1.0(2,8)]nona-3,6-dien-9-one & 1390 & 0.600 \\
10 & 23.958 & 1-Methylindolizine & 0.361 \\
\hline
\end{tabular}

TABLE 4: Relative content (\%) of unique compounds in anhydrous ethanol.

\begin{tabular}{lcccc}
\hline No. & Rt $(\mathrm{min})$ & Compound & Retention index & Relative content (\%) \\
\hline 1 & 5.142 & Thiophene & 766 & 0.064 \\
2 & 6.488 & 2-Methylpyrazine & 923 & 0.137 \\
3 & 9.811 & 2,3-Dimethyl-1H-pyrrole & 968 & 0.155 \\
4 & 11.170 & 6-Methyl-6-ethylfulvene & 1014 & 0.191 \\
5 & 12.724 & Acrylamide & 1080 & 0.098 \\
6 & 14.877 & (-)-Camphor & 1083 & 0.477 \\
7 & 14.974 & 2-Methyl-1-phenylpropene & 1105 & 0.137 \\
8 & 15.691 & 4-Pyridinol & 1150 & 0.157 \\
9 & 17.059 & 3-Phenyl-1,2-butadiene & 1165 & 0.649 \\
10 & 17.518 & Benzo[2,3]bicyclo[3.1.0]hexane & 1272 & 0.188 \\
11 & 20.713 & Citral & 1284 & 0.283 \\
12 & 21.053 & 4-Methyl-2H-benzopyrane & 1330 & 0.160 \\
13 & 22.331 & 1,7-Dimethylnaphthalene & 1432 & 0.224 \\
14 & 25.053 & 2,3,6-Trimethylnaphthalene & 1593 & 0.123 \\
15 & 29.065 & (Z)-8-Hexadecene & 1900 & 0.393 \\
16 & 35.803 & Nonadecane & 1968 & 0.118 \\
17 & 37.154 & Dibutyl phthalate & 0.425 \\
\hline
\end{tabular}

TABLE 5: Relative content (\%) of unique compounds in water.

\begin{tabular}{|c|c|c|c|c|}
\hline No. & Rt (min) & Compound & Retention index & Relative content (\%) \\
\hline 1 & 4.688 & Methallyl cyanide & 741 & 0.065 \\
\hline 2 & 5.083 & Cyclopentanone & 763 & 0.730 \\
\hline 3 & 5.558 & Tetrachloroethylene & 789 & 0.076 \\
\hline 4 & 6.010 & 4-Aminopyridine & 808 & 0.470 \\
\hline 5 & 6.479 & 2-Methylcyclopentanone & 823 & 0.375 \\
\hline 6 & 6.549 & 4-Methylpentanenitrile & 825 & 0.314 \\
\hline 7 & 6.679 & (R)-(+)-3-Methylcyclopentanone & 829 & 0.150 \\
\hline 8 & 7.870 & 2,6-Dimethylpyridine & 867 & 0.596 \\
\hline 9 & 8.091 & Cyclohexanone & 874 & 0.378 \\
\hline 10 & 8.420 & 5,5-Dimethyl-1,3-hexadiene & 885 & 0.079 \\
\hline 11 & 8.816 & 2-Ethylpyrazin & 898 & 0.426 \\
\hline 12 & 8.975 & 2,3-Dimethylpyrazine & 903 & 0.126 \\
\hline 13 & 10.000 & 2,5-Dimethylpyridine & 933 & 0.497 \\
\hline 14 & 10.378 & 5-Methylfurfural & 944 & 1.092 \\
\hline 15 & 11.178 & Phenetole & 968 & 0.185 \\
\hline 16 & 11.346 & 1-Isopropylcyclopentene & 973 & 0.312 \\
\hline 17 & 12.121 & 2-Ethyl-6-methylpyridine & 996 & 0.438 \\
\hline 18 & 12.400 & 2-Ethyl-5-methylpyridine & 1004 & 0.282 \\
\hline 19 & 12.687 & 5-Ethyl-2-methylpyridine & 1013 & 0.207 \\
\hline 20 & 12.743 & 2-Acetyl-5-methylfuran & 1015 & 0.095 \\
\hline 21 & 13.570 & 1-Acetyl-2-methyl-1-cyclopentene & 1040 & 0.378 \\
\hline 22 & 14.229 & 2-Methyl-6-methylene-2,7-octadien-4-ol & 1060 & 0.540 \\
\hline 23 & 14.663 & Sabinene hydrate & 1074 & 0.750 \\
\hline 24 & 14.803 & p-Tolunitrile & 1078 & 0.511 \\
\hline
\end{tabular}


TABLE 5: Continued.

\begin{tabular}{lcccc}
\hline No. & Rt $(\mathrm{min})$ & Compound & Retention index & Relative content (\%) \\
\hline 25 & 15.168 & 2-Methylbenzoxazole & 1089 & 0.568 \\
26 & 15.419 & 2,6-Dimethylphenol & 1103 & 0.820 \\
27 & 15.601 & Phenylacetone & 1106 & 0.116 \\
28 & 15.698 & 1-Isopropyl-1-cyclohexene & 1117 & 0.226 \\
29 & 16.045 & 4-Ethylphenol & 1131 & 0.681 \\
30 & 16.490 & Decamethylcyclopentasiloxane & 1139 & 0.372 \\
31 & 16.721 & Endo-borneol & 1145 & 2.029 \\
32 & 16.918 & 2-Acetyltoluene & 1150 & 0.314 \\
33 & 17.065 & (-)-Terpinen-4-ol & 1156 & 1163 \\
34 & 17.262 & 1-(3-Methylphenyl)ethanone & 1167 & 0.220 \\
35 & 17.460 & (-)-Alpha-terpineol & 1179 & 1181 \\
36 & 17.581 & (+)-Dihydrocarvone & 1189 & 0.318 \\
37 & 17.960 & (+/-)-cis-piperitol & 1201 & 0.338 \\
38 & 18.027 & Verbenone & 1223 & 0.190 \\
39 & 18.279 & (-)-cis-carveol & 1282 & 0.357 \\
40 & 18.610 & 2,4-Dimethylanisole & 1294 & 0.248 \\
41 & 19.293 & Piperitone & 1336 & 0.187 \\
42 & 20.994 & 1-Methylindan-2-one & 1366 & 0.261 \\
43 & 21.356 & Dodecamethylcyclohexasiloxane & 1379 & 0.193 \\
44 & 22.485 & 3,3-Dimethyl-1-indanone & 1468 & 0.233 \\
45 & 23.314 & Methyl eugenol & 0.229 \\
46 & 23.669 & 2,3-Dimethylnaphthalene & & 0.146 \\
47 & 26.001 & 2,4-Di-tert-butylphenol & 0.644 \\
\hline
\end{tabular}

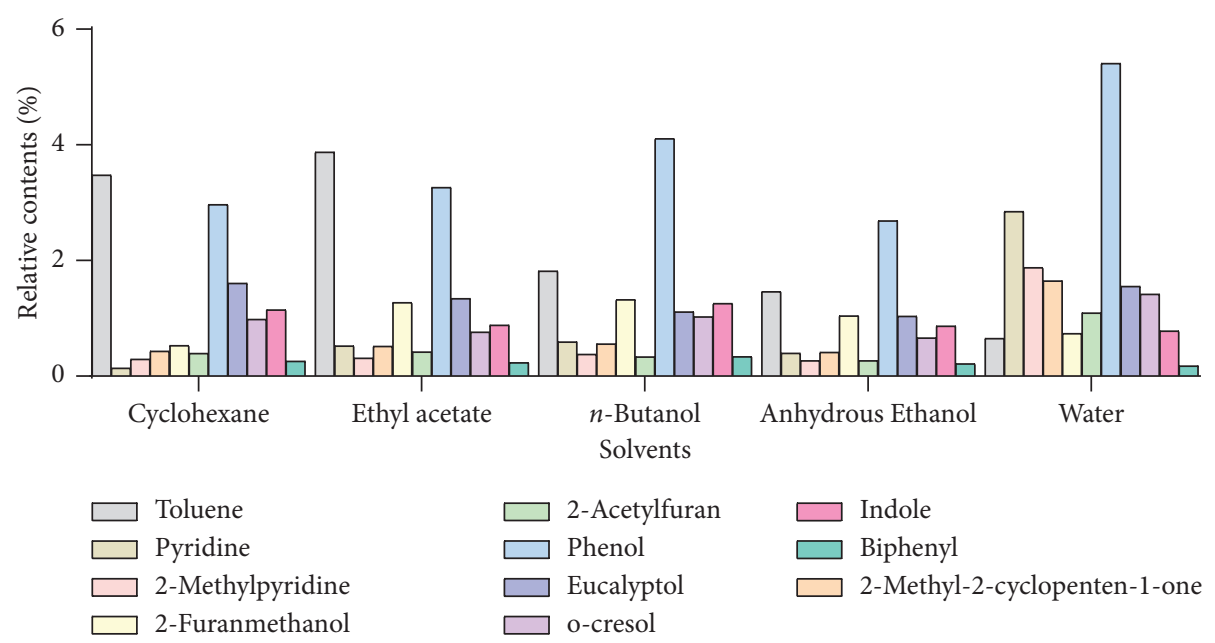

FIgURE 3: Relative contents (\%) of common compounds in the five solvents.

become greater concern. The question of whether moxa smoke is harmless or not has become key to restricting the use of moxibustion.

At present, most studies on moxa smoke have shown that it has many pharmacological effects. A study [27] showed that the superoxide anion scavenging activity of moxa smoke was superoxide dismutase $24.4 \mathrm{U} / \mathrm{mg}$, which was slightly higher than that of partially purified moxa extract and alkali-lignin, but lower than that of sodium ascorbate, gallic acid, and catechin, which further confirmed the antioxidant and pro-oxidative effects of moxa smoke. The methanol extract of moxa smoke has the functions of antioxidation and eliminates free radicals [28]. Another study demonstrated that moxa smoke can improve sperm concentration and promote sperm movement in rats [29].
Although it was suggested that the toxic compounds in moxa smoke were harmful to the human heart, liver, and kidneys, low and middle concentrations had no effects. Moxa smoke at higher concentrations might destroy heart, liver, and kidney function. In fact, it has been reported that moxa smoke can cause related symptoms, such as eustachian tube and throat itching, eye pain, tonsil enlargement, and other symptoms [30-33]. Tar contains two-tenths of a million of a kind of thick cyclic aromatic hydrocarbon called benzo(a) pyrene, which is a strong carcinogen [34]. In a few cases, patients undergoing moxibustion treatment or after treatment had erythema, blisters, and other hypersensitive symptoms, and these conditions disappeared after leaving the moxa smoke environment [35-37]. Research results show that moxibustion may have a greater impact on some 
TABle 6: Toxic compounds in moxa smoke.

\begin{tabular}{|c|c|c|c|c|}
\hline No. & Compound & CAS RN & Chemical ID & PubChem CID \\
\hline 1 & Toluene & $108-88-3$ & D014050 & 1140 \\
\hline 2 & Pyrimidine & $289-95-2$ & C030986 & 9260 \\
\hline 3 & 1-Methylpyrrole & $96-54-8$ & C096654 & 7304 \\
\hline 4 & Pyridine & $110-86-1$ & $\mathrm{C} 023666$ & 1049 \\
\hline 5 & Cyclopentanone & $120-92-3$ & C007201 & 8452 \\
\hline 6 & 1-Octene & $111-66-0$ & $\mathrm{C} 037690$ & 8125 \\
\hline 7 & Tetrachloroethylene & $127-18-4$ & D013750 & 31373 \\
\hline 8 & Octane & $111-65-9$ & $\mathrm{C} 026728$ & 356 \\
\hline 9 & 4-Aminopyridine & $504-24-5$ & D015761 & 1727 \\
\hline 10 & Ethylbenzene & $100-41-4$ & C004912 & 7500 \\
\hline 11 & Styrene & $100-42-5$ & D020058 & 7501 \\
\hline 12 & $p$-Xylene & $106-42-3$ & $\mathrm{C} 031286$ & 7809 \\
\hline 13 & Furfural & $98-01-1$ & D005662 & 7362 \\
\hline 14 & 2,5-Dimethylpyrrole & $625-84-3$ & $\mathrm{C} 067286$ & 12265 \\
\hline 15 & 2-Furanmethanol & $98-00-0$ & C012986 & 7361 \\
\hline 16 & 2-Acetylfuran & $1192-62-7$ & C039669 & 14505 \\
\hline 17 & 3-Methylpyridine & $108-99-6$ & C053603 & 7970 \\
\hline 18 & 2,6-Dimethylpyridine & $108-48-5$ & C013093 & 7937 \\
\hline 19 & o-Xylene & $95-47-6$ & $\mathrm{C} 026114$ & 7237 \\
\hline 20 & Butyrolactone & $96-48-0$ & D015107 & 7302 \\
\hline 21 & Cyclohexanone & $108-94-1$ & C036468 & 7967 \\
\hline 22 & Phenyl ethyne & $536-74-3$ & C044736 & 10821 \\
\hline 23 & m-Xylene & $108-38-3$ & C031285 & 7929 \\
\hline 24 & Propyl benzene & $103-65-1$ & C024268 & 7668 \\
\hline 25 & Nonane & $111-84-2$ & $\mathrm{C} 017573$ & 8141 \\
\hline 26 & 2-Ethylpyridine & $100-71-0$ & C051672 & 7523 \\
\hline 27 & Benzaldehyde & $100-52-7$ & $\mathrm{C} 032175$ & 240 \\
\hline 28 & Cumene & $98-82-8$ & C015763 & 7406 \\
\hline 29 & 2,4-Dimethylpyridine & $108-47-4$ & C078448 & 7936 \\
\hline 30 & Benzofuran & $271-89-6$ & C105430 & 9223 \\
\hline 31 & 5-Methylfurfural & $620-02-0$ & C048065 & 12097 \\
\hline 32 & Phenol & $108-95-2$ & D019800 & 996 \\
\hline 33 & Limonene & $138-86-3$ & D000077222 & 22311 \\
\hline 34 & 3-Ethyltoluene & $620-14-4$ & C029719 & 12100 \\
\hline 35 & 1,2,3-Trimethylbenzene & $526-73-8$ & C010179 & 10686 \\
\hline 36 & Mesitylene & $108-67-8$ & C010219 & 7947 \\
\hline 37 & Phenetole & $103-73-1$ & C079413 & 7674 \\
\hline 38 & Eucalyptol & $470-82-6$ & D000077591 & 2758 \\
\hline 39 & Benzene & $71-43-2$ & D001554 & 241 \\
\hline 40 & Aniline & $62-53-3$ & $\mathrm{C} 023650$ & 6115 \\
\hline 41 & Alpha-methyl styrene & $98-83-9$ & C017915 & 7407 \\
\hline 42 & Butyl butyrate & $109-21-7$ & $\mathrm{C} 022793$ & 7983 \\
\hline 43 & Decane & $124-18-5$ & C012867 & 15600 \\
\hline 44 & Gamma-terpinene & $99-85-4$ & C018669 & 7461 \\
\hline 45 & Alpha-phellandrene & $99-83-2$ & C005403 & 7460 \\
\hline 46 & 5-Ethyl-2-methylpyridine & $104-90-5$ & C019196 & 7728 \\
\hline 47 & o-Cresol & $95-48-7$ & C034047 & 335 \\
\hline 48 & Acrylamide & $79-06-1$ & D020106 & 6579 \\
\hline 49 & 2-Acetyl-5-methylfuran & $1193-79-9$ & C057528 & 14514 \\
\hline 50 & p-Cresol & $106-44-5$ & C032538 & 2879 \\
\hline 51 & 1,2,4-trimethylbenzene & $95-63-6$ & $\mathrm{C} 010313$ & 7247 \\
\hline 52 & o-Cymene & $527-84-4$ & C046257 & 10703 \\
\hline 53 & p-Cymene & $99-87-6$ & $\mathrm{C} 007210$ & 7463 \\
\hline 54 & Guaiacol & $90-05-1$ & D006139 & 460 \\
\hline 55 & m-Cresol & $108-39-4$ & C042041 & 342 \\
\hline 56 & Allylbenzene & $300-57-2$ & $\mathrm{C} 102347$ & 9309 \\
\hline 57 & Indene & $95-13-6$ & C093581 & 7219 \\
\hline 58 & Acetophenone & $98-86-2$ & C038699 & 7410 \\
\hline 59 & Methyl benzoate & $93-58-3$ & $\mathrm{C} 044605$ & 7150 \\
\hline 60 & 2,6-Dimethylphenol & $576-26-1$ & C036531 & 11335 \\
\hline
\end{tabular}


TABle 6: Continued.

\begin{tabular}{|c|c|c|c|c|}
\hline No. & Compound & CAS RN & Chemical ID & PubChem CID \\
\hline 61 & Undecane & $1120-21-4$ & $\mathrm{C} 022884$ & 14257 \\
\hline 62 & Phenylacetone & $103-79-7$ & C008863 & 7678 \\
\hline 63 & 4-Pyridinol & $626-64-2$ & C534143 & 12290 \\
\hline 64 & Naphthalene & $91-20-3$ & $\mathrm{C} 031721$ & 931 \\
\hline 65 & Azulene & $275-51-4$ & C005525 & 9231 \\
\hline 66 & 4-Ethylphenol & $123-07-9$ & $\mathrm{C} 042291$ & 31242 \\
\hline 67 & 4-Allyltoluene & $3333-13-9$ & C092903 & 76851 \\
\hline 68 & Indolizine & $274-40-8$ & $\mathrm{C} 035094$ & 9230 \\
\hline 69 & Phenyl acetonitrile & $140-29-4$ & $\mathrm{C} 006725$ & 8794 \\
\hline 70 & 2,3-Dimethylphenol & $526-75-0$ & $\mathrm{C} 054067$ & 10687 \\
\hline 71 & 1,2,3,4-Tetramethylbenzene & $488-23-3$ & C021246 & 10263 \\
\hline 72 & (-)-Alpha-terpineol & $10482-56-1$ & $\mathrm{C} 016775$ & 443162 \\
\hline 73 & 3,5-Dimethylphenol & $108-68-9$ & C016834 & 7948 \\
\hline 74 & Terpinen-4-ol & $562-74-3$ & C034019 & 11230 \\
\hline 75 & Dodecane & $112-40-3$ & C007548 & 8182 \\
\hline 76 & Catechol & $120-80-9$ & C034221 & 289 \\
\hline 77 & 5,6-Dimethylbenzimidazole & $582-60-5$ & C015158 & 675 \\
\hline 78 & Tridecane & $629-50-5$ & C094074 & 12388 \\
\hline 79 & (E)-Cinnamaldehyde & $104-55-2$ & $\mathrm{C} 012843$ & 637511 \\
\hline 80 & Indole & $120-72-9$ & $\mathrm{C} 030374$ & 798 \\
\hline 81 & Isoquinoline & $119-65-3$ & C039109 & 8405 \\
\hline 82 & Citral & $5392-40-5$ & C007076 & 638011 \\
\hline 83 & Hydroquinone & $123-31-9$ & C031927 & 785 \\
\hline 84 & Biphenyl & $92-52-4$ & C010574 & 7095 \\
\hline 85 & 1-Tridecene & $2437-56-1$ & $\mathrm{C} 028691$ & 17095 \\
\hline 86 & Tetradecane & $629-59-4$ & $\mathrm{C} 024713$ & 12389 \\
\hline 87 & 2-Methylnaphthalene & $91-57-6$ & $\mathrm{C} 027384$ & 7055 \\
\hline 88 & 1-Methylnaphthalene & $90-12-0$ & $\mathrm{C} 025968$ & 7002 \\
\hline 89 & 2-Methoxy-4-vinylphenol & $7786-61-0$ & C014245 & 332 \\
\hline 90 & 2,6-Dimethylnaphthalene & $581-42-0$ & $\mathrm{C} 028519$ & 11387 \\
\hline 91 & 2-Methylhydroquinone & $95-71-6$ & $\mathrm{C} 062397$ & 7253 \\
\hline 92 & Methyl eugenol & $93-15-2$ & $\mathrm{C} 005223$ & 7127 \\
\hline 93 & 5-Methylindole & $614-96-0$ & C093726 & 11978 \\
\hline 94 & 2,3-Dimethylnaphthalene & $581-40-8$ & C091753 & 11386 \\
\hline 95 & 3-Methylindole & $83-34-1$ & D012862 & 6736 \\
\hline 96 & 1,4-Dimethylnaphthalene & $571-58-4$ & C031969 & 11304 \\
\hline 97 & 2,4-Di-tert-butylphenol & $96-76-4$ & C056559 & 7311 \\
\hline 98 & Dibenzofuran & $132-64-9$ & $\mathrm{C} 023614$ & 568 \\
\hline 99 & Phenylephrine & $59-42-7$ & D010656 & 6041 \\
\hline 100 & Heptadecane & $629-78-7$ & $\mathrm{C} 016486$ & 12398 \\
\hline 101 & Spathulenol & $6750-60-3$ & C013258 & 92231 \\
\hline 102 & 1-Octadecene & $112-88-9$ & C109760 & 8217 \\
\hline 103 & Chamazulene & $529-05-5$ & $\mathrm{C} 013872$ & 10719 \\
\hline 104 & Phenanthrene & $85-01-8$ & $\mathrm{C} 031181$ & 995 \\
\hline 105 & Octadecane & $593-45-3$ & $\mathrm{C} 022883$ & 11635 \\
\hline 106 & Pinane & $473-55-2$ & C030216 & 10129 \\
\hline 107 & Nonadecane & $629-92-5$ & $\mathrm{C} 061580$ & 12401 \\
\hline 108 & Hentriacontane & $630-04-6$ & C049203 & 12410 \\
\hline 109 & Methyl palmitate & $112-39-0$ & C019012 & 8181 \\
\hline 110 & Ambrettolide & $123-69-3$ & $\mathrm{C} 008563$ & 5365703 \\
\hline 111 & Dibutyl phthalate & $84-74-2$ & D003993 & 3026 \\
\hline 112 & Icosane & $112-95-8$ & C050821 & 8222 \\
\hline
\end{tabular}

people with chronic pharyngitis, leading to coughing due to moxa smoke allergy, but these symptoms gradually improved after ventilation [38]. Some scholars have placed rats in a dynamic exposure cabinet and observed the content of
Ox-LDL in their serum. The results showed that the content of Ox-LDL decreased gradually with the increase of moxa smoke concentration, suggesting that moxa smoke can reduce the degree of platelet aggregation. Therefore, it may 


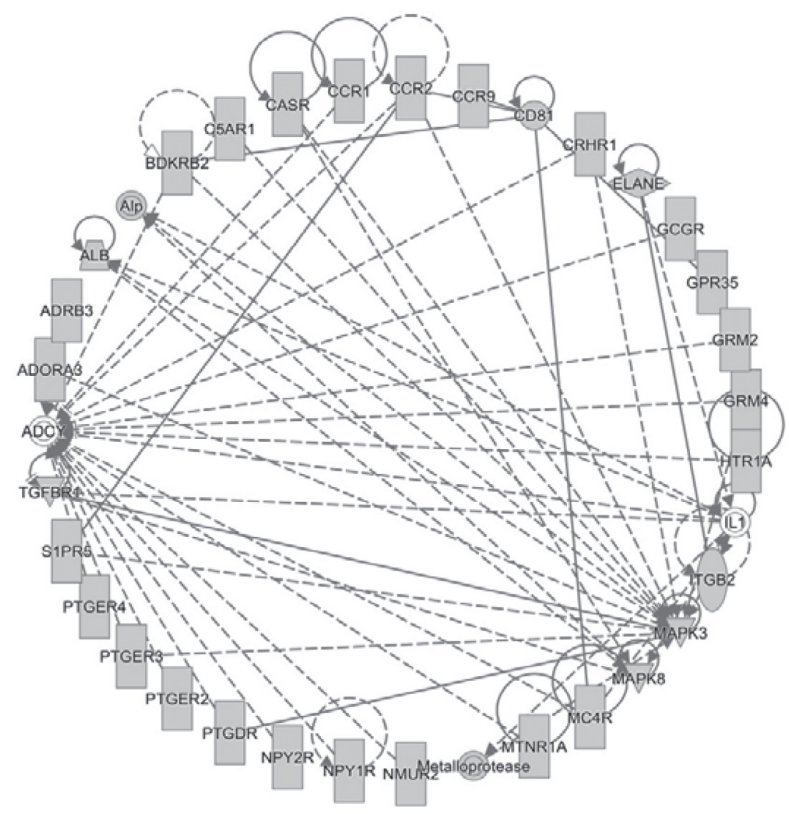

FIgURE 4: Molecular network with a maximum score of 43. Each node in the figure represents 1 molecule, the solid lines represent a direct interaction between two molecules, and the dotted lines represent an indirect interaction between two molecules.

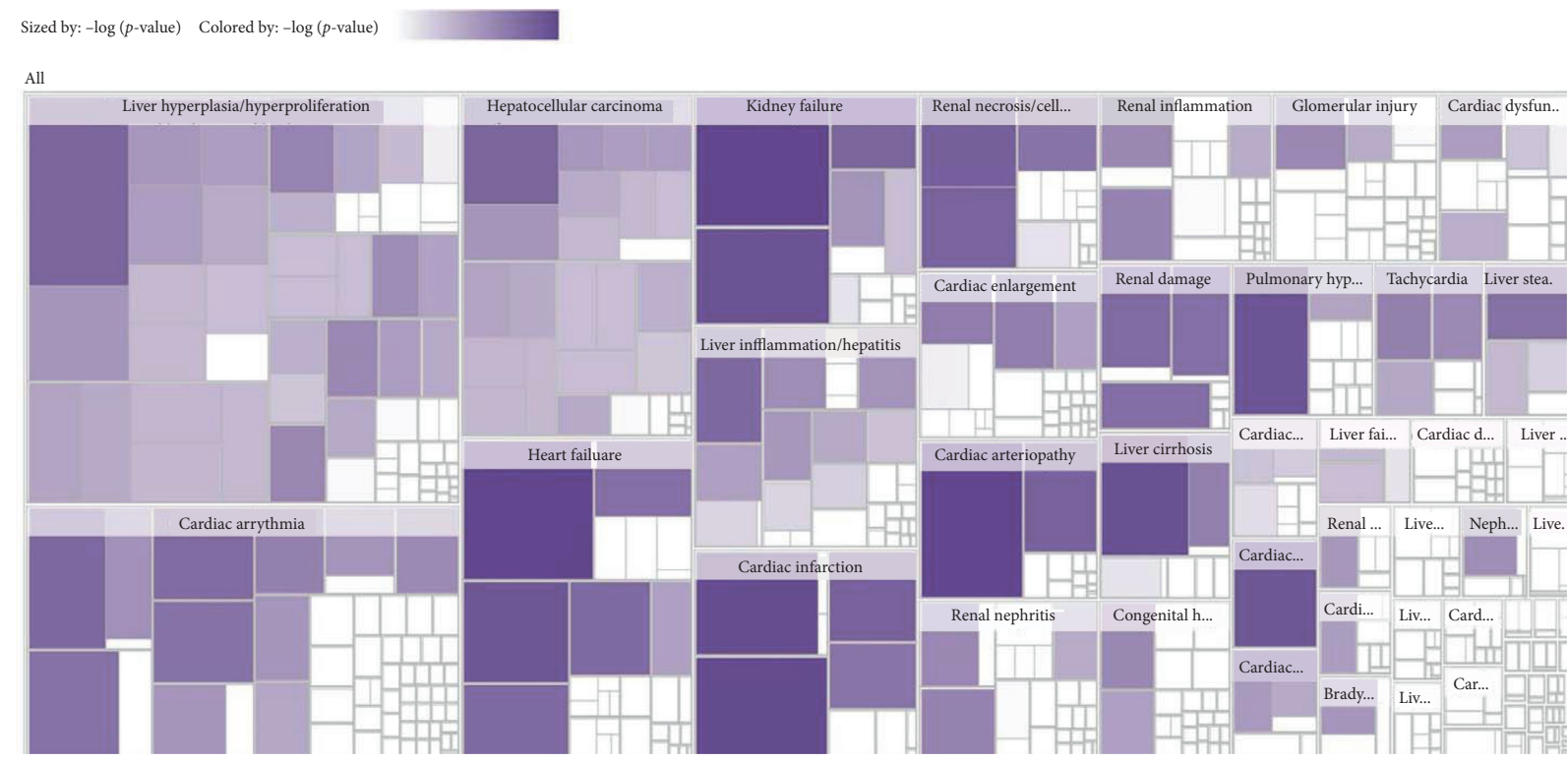

FIGURE 5: A heat map of the biological pathway of toxic compounds. The pathway scores are displayed using a purple color gradient, where darker purple corresponds to higher scores (increased statistical significance).

improve microcirculation and promote metabolism of the body. Low concentrations of moxa smoke have no noticeable damage to vascular endothelium, while medium concentrations can cause a certain degree of vascular endothelium damage $[39,40]$.

The moxa sticks were encased in $A$. argyi floss, which is made of dried $A$. argyi leaves. There have been many experimental studies on the toxicity of $A$. argyi, which were not limited to conventional acute toxicity, subacute toxicity, or chronic toxicity. Domestic scholars have conducted in-depth studies on the hepatorenal toxicity, embryonic toxicity, and genetic toxicity caused by $A$. argyi. The research objects were not limited to the whole animal, but also extended to the cellular level, and the intrinsic mechanism of some toxicity of $A$. argyi was also discussed. The relationship between quantity, time and toxicity, and a safe time span for use were also discussed. However, some of the results showed that $A$. argyi had hepatotoxicity, especially the essential oil of A. argyi $[41,42]$. The dosage of $A$. argyi or moxa sticks used in toxic experiments was more than 10 to 200 times the clinical dosage. According to the results of this paper, we carried out toxicological experiment of moxa smoke in rats. We followed the steps outlined in our patent, "A device for enriching moxa smoke and its analytical method," patent 
number: CN202010327163.6 [43]. Rats exposed to $756650 \mathrm{mg} / \mathrm{m}^{3}$ concentration of moxa smoke (concentration of moxa smoke in 50 moxa sticks) were compared with the control group, and the structure of myocardial cell, hepatic cell, and the renal tubules showed changes (Supplementary Figure S1) such as cardiac hypertrophy, degeneration and necrosis, and dilatation of renal tubules, respectively.

In a word, we should not discuss the toxicity in terms of toxicity in isolation but should comprehensively consider the clinical use characteristics of traditional Chinese medicine. However, in clinical application, we should pay attention to its "toxicity" to human body and try to avoid overuse. Therefore, moxibustion rooms should have installed ventilation equipment or the room should have adequate artificial ventilation so that the health of patients and practitioners can be guaranteed. The safety of compounds in moxa smoke needs to be further studied. The results of this study provide a basis for a safety evaluation of moxa smoke in the future.

\section{Data Availability}

The data used to support the findings of this study are included within the article and in the supplementary figure. The prior studies (and datasets) are cited at relevant places within the text as references [21, 43].

\section{Conflicts of Interest}

The authors declare that they have no conflicts of interest.

\section{Authors' Contributions}

L.H.N. conceptualized the study. S.S. and X.X.Y. contributed to methodology and formal analysis, validated the study, and wrote, reviewed, and edited the manuscript. W.W.L. provided software. X.X.Y. wrote and prepared the original draft. L.H.N was responsible for project administration. All authors have read and agreed to the published version of the manuscript.

\section{Acknowledgments}

This work was financially subsidized by the 2018 First Class Discipline Construction Project of Jiangxi Province (no. JXSYLXK-ZHYAO145). The authors thank LetPub (http:// www.letpub.com) for its linguistic assistance during the preparation of this manuscript.

\section{Supplementary Materials}

Supplementary Figure S1: microscopic observations of heart, liver, and kidney pathology. (Supplementary Materials)

\section{References}

[1] M. Y. Lim, J. Huang, and B. Zhao, "Standardisation of moxibustion: challenges and future development," Acupuncture in Medicine, vol. 33, no. 2, pp. 142-147, 2015.

[2] M. S. Lee, T.-Y. Choi, J. W. Kang, B.-J. Lee, and E. Ernst, "Moxibustion for treating pain: a systematic review," The
American Journal of Chinese Medicine, vol. 38, no. 5, pp. 829-838, 2010.

[3] C. Zhou, X. Feng, J. Wang et al., "Research advance on moxa smoke," Journal of Acupuncture and Tuina Science, vol. 9, no. 2, pp. 67-72, 2011.

[4] J. Wu, "Research progress on mechanism and safety of moxibustion," Chinese Folk Therapy, vol. 27, pp. 105-107, 2019.

[5] Y. Lu, C. M. Ye, J. F. Gao et al., "Moxa fumigation for 575 surgical infectious conditions (annex: inhibiting bacteria test of the moxa smoke)," An Hui Zhong Yi Xue Yuan Xue Bao, vol. 7, no. 4, pp. 36-37, 1998.

[6] K. Cheng, M. M. Luo, H. Q. Wang et al., "Research progress on the correlation of artemisinin," Journal of Practical Chinese Medicine, vol. 35, pp. 632-634, 2019.

[7] N. Hitosugi, R. Ohno, I. Hatsukari et al., "Diverse biological activities of moxa extract and smoke," In Vivo (Athens, Greece), vol. 15, no. 3, pp. 249-254, 2001.

[8] J. K. Anastasi, D. J. Mcmahon, and G. H. Kim, "Symptom management for irritable bowel syndrome," Gastroenterology Nursing, vol. 32, no. 4, pp. 243-255, 2009.

[9] D. J. Stein, "Massage acupuncture, moxibustion, and other forms of complementary and alternative medicine in inflammatory bowel disease," Gastroenterology Clinics of North America, vol. 46, no. 4, pp. 875-880, 2017.

[10] S. Yamaguchi and N. Araki, "The practical use of acupuncture and moxibustion treatment cooperated with neurological practice," Rinsho Shinkeigaku, vol. 52, no. 11, pp. 1287-1289, 2012.

[11] H. Xu, B. Zhao, Y. Cui et al., "Effects of moxa smoke on monoamine neurotransmitters in SAMP8 mice," EvidenceBased Complementary and Alternative Medicine, vol. 2013, Article ID 178067, 6 pages, 2013.

[12] Y. U. Chang, W. Xiang, and Y. Hui, "Small sample size epidemiological survey of the time-related exposure reaction of acupuncturists under the moxa-smoke environment," Journal of Chengdu University of Traditional Chinese Medicine, vol. 38, no. 4, pp. 6-9, 2015.

[13] X. Wang, C. Yu, H. Yang et al., "Symptoms of long-term exposure to moxa smoke in acupuncturists: a correlation analysis," Journal of Traditional Chinese Medicine/Chung I Tsa Chih Ying Wen pan, vol. 38, no. 1, pp. 132-138, 2018.

[14] R. Zhang, "Accidents in acupuncture treatment: history and current state," Journal of Chinese Integrative Medicine, vol. 2, no. 4, pp. 306-313, 2004.

[15] O. S. Kwon, S. J. Cho, K.-H. Choi et al., "Safety recommendations for moxa use based on the concentration of noxious substances produced during commercial indirect moxibustion," Acupuncture in Medicine, vol. 35, no. 2, pp. 93-99, 2017.

[16] F. Mo, C. Chi, M. Guo, X. Chu, Y. Li, and X. Shen, "Characteristics of selected indoor air pollutants from moxibustion," Journal of Hazardous Materials, vol. 270, pp. 53-60, 2014.

[17] A. P. Davis, B. L. King, S. Mockus et al., "The comparative toxicogenomics database: update 2011," Nucleic Acids Research, vol. 39, pp. 1104-1114, 2013.

[18] A. P. Davis, C. J. Grondin, R. J. Johnson et al., "The comparative toxicogenomics database: update 2017," Nucleic Acids Research, vol. 45, no. D1, pp. 972-978, 2016.

[19] L. Gong, D. Wang, L. Zhang, X. Xie, H. Sun, and J. Gu, "Genetic changes in rat proximal nerve stumps after sciatic nerve transection," Annals of Translational Medicine, vol. 7, no. 23, p. 763, 2019. 
[20] A. Krämer, J. Green, J. Pollard Jr., and S. Tugendreich, "Causal analysis approaches in ingenuity pathway analysis," Bioinformatics, vol. 30, no. 4, pp. 523-530, 2014.

[21] H. N. Liu, S. Shan, X. J. Yan et al., "A method of using Terahertz wave to detect the quality of moxa column," CN110793933B, 2020.

[22] T. Mohammad, S. Batra, R. Dahiya et al., "Identification of high-affinity inhibitors of cyclin-dependent kinase 2 towards anticancer therapy," Molecules, vol. 24, no. 24, pp. 4589-4601, 2019.

[23] L. Barberini, A. Restivo, A. Noto et al., "A gas chromatographymass spectrometry (GC-MS) metabolomic approach in human colorectal cancer (CRC): the emerging role of monosaccharides and amino acids," Annals of Translational Medicine, vol. 7, no. 23, p. 727, 2019.

[24] K. Karthika, G. Gargi, S. Jamuna et al., "The potential of antioxidant activity of methanolic extract of Coscinium fenestratum (Goetgh.) Colebr (Menispermaceae)," Saudi Journal of Biological Sciences, vol. 26, no. 5, pp. 1037-1042, 2018.

[25] W. Duan, F. Meng, H. Cui, Y. Lin, G. Wang, and J. Wu, "Ecotoxicity of phenol and cresols to aquatic organisms: a review," Ecotoxicology and Environmental Safety, vol. 157, pp. 441-456, 2018.

[26] Z. Yao, B. P. Cary, C. A. Bingman et al., "Use of a stereochemical strategy to probe the mechanism of phenol-soluble modulin $\alpha 3$ toxicity," Journal of the American Chemical Society, vol. 141, no. 19, pp. 7660-7664, 2019.

[27] H. Matsumoto, J. Shimada, H. Nagasaka et al., "Inhibition by Moxa smoke of NO production and iNOS expression in mouse macrophage-like cells raw 264.7," In Vivo, vol. 19, no. 2, pp. 471-474, 2005.

[28] B.-X. Zhao, P. Liu, C. X. Huang et al., "A toxicological investigation of the air quality in a moxibustion treatment room as measured through particulate concentration and oxidative capacity," World Journal of Traditional Chinese Medicine, vol. 1, no. 2, pp. 61-67, 2015

[29] L. Wang, L. Han, P. Liu et al., "Moxa smoke: is it a beneficial or hazardous factor for infertility? A preclinical study on sperm parameters and sex hormones in male rats," European Journal of Integrative Medicine, vol. 8, no. 1, pp. 28-35, 2016.

[30] R. He, L. Han, P. Liu et al., "Lung function decline after 24 Weeks of moxa smoke exposure in rats," Evidence-based Complementary and Alternative Medicine, vol. 2019, Article ID 9236742, 7 pages, 2019.

[31] B. Q. Ji, J. Li, N. X. Zhao, and R. Qin-you, "Pulmonary function changes of 80 healthy young people after acupuncture or moxibustion treatment on Feishu," Journal of Nanjing University of Traditional Chinese Medicine, vol. 18, pp. 174-175, 2002.

[32] Y. X. Cui, B. X. Zhao, H. Yu et al., "Effects of moxa (Folium artemisiae argyi) smoke exposure on heart rate and heart rate variability in healthy young adults: a randomized, controlled human study," Evidence-Based Complementray and Alternative Medicine, vol. 2013, Article ID 510318, 10 pages, 2013.

[33] H. Sakagami, H. Matsumoto, K. Satoh et al., "Cytotoxicity and radical modulating activity of moxa smoke," In Vivo, vol. 19, no. 2, pp. 391-397, 2005.

[34] C. Gong, L. Qi, Y. Huo et al., "Anticancer effect of Limonin against benzo(a)pyrene-induced lung carcinogenesis in Swiss albino mice and the inhibition of A549 cell proliferation through apoptotic pathway," Journal of Biochemical and Molecular Toxicology, vol. 33, no. 12, p. e22374, 2019.

[35] X. Q. Li and M. Liu, "One case study of allergic reaction to moxibustion," Zhong Guo Zhen Jiu, vol. 20, no. 1, p. 63, 2000.
[36] Z. Y. Feng and J. An, "One case study of allergic reaction to moxibustion," Zhong Guo Zhen Jiu, vol. 25, no. 12, p. 899, 2005.

[37] H. X. Li and S. W. Liu, "Two cases studies of allergic reaction to moxibustion," Zhong Guo Zhong Yi Ji Zheng, vol. 17, no. 6, pp. 859-860, 2008.

[38] J.-E. Park, S.-S. Lee, M. S. Lee, S.-M. Choi, and E. Ernst, "Adverse events of moxibustion: a systematic review," Complementary Therapies in Medicine, vol. 18, no. 5, pp. 215-223, 2010.

[39] J. H. Lee, "How does moxibustion possibly work?" EvidenceBased Complementary and Alternative Medicine, vol. 2013, Article ID 198584, 8 pages, 2013.

[40] J. T. Liu, B. X. Zhao, Y. X. Cui et al., "Effects of shenque moxibustion on behavioral changes and brain oxidative state in apolipoprotein E-deficient mice," Evidence-Based Complementary and Alternative Medicine, vol. 2015, Article ID 804804, 8 pages, 2015.

[41] H. J. Liu, H. Q. Dong, S. Zhan et al., "Metabonomics study on acute hepatotoxicity mechanism of volatile oil from Artemisia argyi based on 1H-NMR," Chinese Journal of Traditional Chinese Medicine, vol. 44, no. 4, pp. 827-832, 2019.

[42] H. J. Liu, T. H. Li, S. Zhan et al., "Acute hepatotoxicity induced by volatile oil from Artemisia argyi and its mechanism," Chinese Journal of Clinical Pharmacology and Therapeutics, vol. 22, no. 3, pp. 248-252, 2017.

[43] S. Shan, H. N. Liu, P. Nie et al., "A device for enriching moxa smoke and its analytical method," CN111366679A, 2020. 